VOLUME LI

JUNE $\cdot 1993$

NUMBER 2

JUNE 1993, PAGES 201-216

\title{
EQUILIBRIA OF THE CIRCULAR ELASTICA UNDER A UNIFORM CENTRAL FORCE FIELD
}

\author{
BY \\ R. W. DICKEY (University of Wisconsin, Madison, Wisconsin) \\ AND
}

J. J. ROSEMAN (Tel-Aviv University, Tel-Aviv, Israel)

\begin{abstract}
A mathematical model for the problem of an inextensible circular elastica under a uniform centrally directed force field is derived and studied. It is shown analytically that stable large amplitude solutions exist at forces $P<P_{1}$, the first eigenpressure for the linearized model, and it is shown numerically that these solutions have only one axis of symmetry. These results agree with experiment. In addition, numerical solutions are calculated for states with more than one axis of symmetry which resemble those found in the literature on elastic rings under hydrostatic pressure.
\end{abstract}

1. Introduction. The purpose of this paper is to discuss the buckled states of an extensible circular elastica under a uniform centrally directed force field. The equations governing the deformation of the circular ring are derived in the appendix (Sec. 5). They are

$$
\frac{d^{2} \psi}{d \theta^{2}}+\lambda \sin \psi-\mu \sin (\psi+\theta)=0,
$$

where $\psi$ is to satisfy the constraint

$$
\int_{0}^{\pi} \cos (\psi+\theta) d \theta=0
$$

and boundary conditions

$$
\psi(0)=\psi(\pi)=0 .
$$

The dependent variable $\psi$ is the change in angle the normal to the center line makes before and after deformation. The parameter $\mu$ is a Lagrange multiplier to be used to satisfy the constraint (1.2) and

$$
\lambda=\frac{P a}{E I}
$$

Received February 12, 1991.

1991 Mathematics Subject Classification. Primary 73H05.

(C)1993 Brown University 
where $P$ is the applied loading, $a$ is the radius of the ring, $E$ is the Young's modulus, and $I$ is a geometry parameter,

$$
I=\iint_{A}\left(\frac{r}{a-r}\right)^{2} d A,
$$

$A$ being the cross section of the ring. The tangential and normal displacements $u$ and $w$ are determined by the pair of linear ordinary differential equations

$$
\begin{gathered}
\frac{w^{\prime}-u}{a}=-\sin \psi, \\
1+\frac{w+u^{\prime}}{a}=\cos \psi,
\end{gathered}
$$

with initial conditions

$$
u(0)=w(0)=0 .
$$

The Eqs. (1.6) and initial conditions (1.3) and (1.7) imply that $u^{\prime}(0)=w^{\prime}(0)=0$.

The buckling of the circular ring under hydrostatic pressure has been studied by Tadjbakhsh and Odeh [1] and Antman [2, 3]. In these papers it is shown that all the buckled states have at least two axes of symmetry and the buckled states found therein occur, in effect, for $\lambda>4$. Stoker [4, pp. 82-83] writes, "Consider first a complete circular arch for which the middle line is a complete circle without ends. Experience shows: as the load $p$ increases from zero, the arch is first deformed into an arch of smaller radius, ... , until a certain critical load occurs as a localized 'dip' in the arch at some point...". Clearly, the buckled state described has only one axis of symmetry. (Stoker does not precisely stipulate the loading, but until the moment when buckling begins, both hydrostatic pressure and a uniform centrally directed force field should produce the deformation which he describes.) The theory described by (1.1), (1.2), and (1.3) has solutions having two or more axes of symmetry, similar to all of those found in [1] and, in addition, there are stable solutions with one axis of symmetry occurring for values of $\lambda<4$.

In Sec. 2, it is shown that the linearized problem has only the trivial solution unless $\lambda=n^{2}, n=2,3, \ldots$. In Sec. 3, a Galerkin type procedure (cf. $\left.[5,6]\right)$ is used to show that the nonlinear problem has a nontrivial solution (with energy less than the energy of the trivial solution) for $\lambda>\Lambda(\Lambda \approx 2.62)$. In Sec. 4 , the nonlinear problem is solved numerically. We find solutions with one axis of symmetry and solutions with two or more axes of symmetry similar to those in [1]. The solutions with one axis of symmetry are obtained for values of $\lambda<4$ (the value at which linear buckling begins) as well as values of $\lambda \geq 4$. The computational results indicate that the solutions with one axis of symmetry actually exist for $\lambda>\Lambda_{0}\left(\Lambda_{0} \approx 1.2\right)$. The bifurcation diagram is drawn and the actual shape of the buckled ring is sketched for various values of $\lambda$.

2. The linearized problem. In this section, we study the linearization of equations (1.1), (1.2), and (1.3). To obtain the linearized system of equations, we first write $\psi(\theta)=\varepsilon \gamma(\theta)$ where $\varepsilon>0$ is a measure of the amplitude, assumed very small. Then 
we substitute into (1.1), (1.2), and (1.3), expand the resulting expressions in power series in $\varepsilon$ and neglect terms of order $\varepsilon^{2}$ and smaller. There results

$$
\begin{gathered}
\frac{d^{2} \gamma}{d \theta^{2}}+\lambda \gamma-\mu \cos \theta \gamma=\frac{\mu}{\varepsilon} \sin \theta, \\
\int_{0}^{\pi} \gamma(\theta) \sin \theta d \theta=0, \\
\gamma(0)=\gamma(\pi)=0,
\end{gathered}
$$

and, in addition, we require that

$$
\gamma(\theta)=O(1) \quad \text { as } \varepsilon \rightarrow 0
$$

in order to be consistent with the physical assumption of very small changes from the unstressed reference state.

When $\mu=0$, it is easily seen that nontrivial solutions for $\gamma$ exist if and only if $\lambda=\lambda_{k}=k^{2}$ with $k=2,3,4, \ldots$, and, for $\lambda=\lambda_{k}, \gamma_{k}(\theta)=C \sin k \theta$ is a solution for all $C$. These solutions correspond to rings having two or more axes of symmetry. However, for $\mu \neq 0$, we shall now demonstrate the following:

Lemma 2.1. For $\mu \neq 0$, the system (2.1)-(2.4) has no solution.

Proof. We shall in fact show that there are no nontrivial solutions in the space $L_{2}(0, \pi)$, and therefore no classical smooth solutions.

We begin by supposing that a solution $\gamma \in L_{2}(0, \pi)$ does exist. Then from (2.1), it follows that $d^{2} \gamma / d \theta^{2} \in L_{2}(0, \pi)$. Since the sequence $\{\sin k \theta\}_{k=1}^{\infty}$ is a basis for $L_{2}(0, \pi)$, the functions $\gamma$ and $d^{2} \gamma / d \theta^{2}$ may be represented as an expansion in these basis functions and, from (2.2) and (2.3), the expansions must be of the form

$$
\begin{gathered}
\gamma(\theta)=\sum_{k=2}^{\infty} B_{k} \sin k \theta, \\
\frac{d^{2} \gamma}{d \theta^{2}}=-\sum_{k=2}^{\infty} k^{2} B_{k} \sin k \theta .
\end{gathered}
$$

(The coefficient $B_{1}$ is equal to zero because of (2.2).) Substituting (2.5) and (2.6) into $(2.1)$ yields

$$
-\sum_{k=2}^{\infty} k^{2} B_{k} \sin k \theta+\lambda \sum_{k=2}^{\infty} B_{k} \sin k \theta-\mu \cos \theta \sum_{k=2}^{\infty} B_{k} \sin k \theta=\frac{\mu}{\varepsilon} \sin \theta
$$

and then, from the identity

$$
2 \cos \theta \sin k \theta=\sin (k+1) \theta+\sin (k-1) \theta
$$

we have, after some obvious changes of index, and with $B_{1}=0$,

$$
\sum_{k=2}^{\infty}\left(\lambda-k^{2}\right) B_{k} \sin k \theta-\frac{\mu}{2} \sum_{k=2}^{\infty} B_{k-1} \sin k \theta-\frac{\mu}{2} \sum_{k=1}^{\infty} B_{k+1} \sin k \theta=\frac{\mu}{\varepsilon} \sin \theta .
$$


By matching coefficients of $\sin k \theta$, we see that, for $k=1$,

$$
-\frac{\mu}{2} B_{2}=\frac{\mu}{\varepsilon}
$$

and so, for $\mu \neq 0$,

$$
B_{2}=-\frac{2}{\varepsilon}
$$

while

$$
\left(k^{2}-\lambda\right) B_{k}+\frac{\mu}{2} B_{k-1}+\frac{\mu}{2} B_{k+1}=0
$$

for $k \geq 2$.

We see that each $B_{k}$ is uniquely determined by the relations (2.9), (2.10). However,

$$
\|\gamma\|=\left[\int_{0}^{\pi} \gamma^{2} d \theta\right]^{1 / 2}=\left[\frac{\pi}{2} \sum_{k=2}^{\infty} B_{k}^{2}\right]^{1 / 2} \geq \sqrt{\frac{\pi}{2}}\left|B_{2}\right|=\frac{\sqrt{2 \pi}}{\varepsilon}
$$

so that, for all $\lambda$ and $\mu \neq 0,\|\gamma\| \rightarrow \infty$ as $\varepsilon \rightarrow 0$, which contradicts (2.4).

From Lemma (2.1) and the previous comments concerning the case when $\mu=0$, we may deduce

LemMA 2.2. For $\lambda<4$ and all values of $\mu$, there exists no nontrivial solution to the system (2.1)-(2.4).

3. Nonlinear theory. In this section we will prove the existence of a nontrivial solution of (1.1), (1.2), and (1.3) for values of $\lambda>2.62$. If the problem (1.1), (1.2), and (1.3) has a sufficiently differentiable solution it can be written as a Fourier series

$$
\psi=\sum_{n=1}^{\infty} a_{n} \sin n \theta
$$

If the series could be differentiated twice, it would follow from (1.1) that

$$
-\sum_{n=1}^{\infty} n^{2} a_{n} \sin n \theta+\lambda \sin \psi-\mu \sin (\psi+\theta)=0,
$$

where $\psi$ is given by (3.1). Multiplying equation (3.2) by $\sin j \theta$ and integrating from $\theta=0$ to $\theta=\pi$ gives an infinite system of nonlinear algebraic equations for the determination of the coefficients $a_{j}$ :

$$
j^{2} a_{j}-\frac{2 \lambda}{\pi} \int_{0}^{\pi} \sin \psi \sin j \theta d \theta+\frac{2 \mu}{\pi} \int_{0}^{\pi} \sin (\psi+\theta) \sin j \theta d \theta=0
$$

for $j=1,2, \ldots$ In addition, the function $\psi$ (cf. (3.1)) must satisfy the constraint (1.2).

In order to prove the existence of a solution to (3.3) satisfying (1.2), it is convenien1 to begin by considering the related finite system:

$$
\begin{aligned}
j^{2} a_{j} & -\frac{2 \lambda}{\pi} \int_{0}^{\pi} \sin \psi_{N} \sin j \theta d \theta \\
& +\frac{2 \mu}{\pi} \int_{0}^{\pi} \sin \left(\psi_{N}+\theta\right) \sin j \theta d \theta=0
\end{aligned}
$$


for $j=1,2, \ldots, N$ with the constraint

$$
C_{N}\left(a_{1}, \ldots, a_{N}\right)=\int_{0}^{\pi} \cos \left(\psi_{N}+\theta\right) d \theta=0
$$

where

$$
\psi_{N}=\sum_{n=1}^{N} a_{n} \sin n \theta
$$

In order to prove the existence of a nontrivial solution to (3.4) satisfying the constraint (3.5), we define a function

$$
H_{N}\left(a_{1}, \ldots, a_{N}\right)=\frac{1}{2} \sum_{n=1}^{N} n^{2} a_{n}^{2}+\frac{2 \lambda}{\pi} \int_{0}^{\pi}\left(\cos \left(\psi_{N}\right)-1\right) d \theta
$$

and consider the problem of minimizing $H_{N}$ over the vectors $\left(a_{1}, \ldots, a_{N}\right)$ satisfying (3.5). We note that $H_{N}(0, \ldots, 0)=0$. Thus, we would like to show that the minimum (if it exists) is negative. For this purpose note

$$
H_{N}\left(a_{1}, 0, \ldots, 0\right)=\frac{1}{2} a_{1}^{2}+\frac{2 \lambda}{\pi} \int_{0}^{\pi}\left(\cos \left(a_{1} \sin \theta\right)-1\right) d \theta,
$$

where (cf. (3.5))

$$
C_{N}\left(a_{1}, 0, \ldots, 0\right)=\int_{0}^{\pi} \cos \left(a_{1} \sin \theta+\theta\right) d \theta=J_{1}\left(-a_{1}\right)=0 .
$$

The function $J_{1}$ is the Bessel function of first order (cf. [9]). Equation (3.8) is satisfied if $a_{1}=j_{1 n}$, i.e., if $a_{1}$ is one of the zeros of $J_{1}$. For the choice $a_{1}=j_{11}$, equation (3.7) becomes

$$
H_{N}\left(j_{11}, 0, \ldots, 0\right)=\frac{1}{2} j_{11}^{2}+2 \lambda\left(J_{0}\left(j_{11}\right)-1\right),
$$

where $J_{0}$ is the Bessel function of order zero. $H_{N}\left(j_{11}, 0, \ldots, 0\right)<0$ if

$$
\lambda>\frac{j_{11}^{2}}{4\left(1-J_{0}\left(j_{11}\right)\right)}=\Lambda \text {. }
$$

The right side of (3.10) is easily evaluated to show that $H_{N}<0$ if $\lambda>2.62$. Thus $H_{N}$ has negative values for $\lambda<4$, the smallest eigenvalue of the linear problem.

Consider the set $M$ of vectors $\left(a_{1}, \ldots, a_{N}\right)$ such that $H_{N}\left(a_{1}, \ldots, a_{N}\right) \leq 0$ and $C_{N}\left(a_{1}, \ldots, a_{N}\right)=0 . M$ is not empty since both $(0, \ldots, 0)$ and $\left(j_{11}, 0, \ldots, 0\right)$ are in $M$. In addition, $M$ is bounded since $H_{N} \leq 0$ implies

$$
\sum_{n=1}^{N} n^{2} a_{n}^{2} \leq \frac{4 \lambda}{\pi} \int_{0}^{\pi}\left(1-\cos \psi_{N}\right) d \theta \leq 8 \lambda .
$$

The boundedness of $M$ is an immediate consequence of (3.11). Since $H_{N}$ is a continuous function of its arguments, $M$, as defined above, is a closed set. Thus, $M$ is compact. 
$H_{N}\left(a_{1}, \ldots, a_{N}\right)$ is a continuous function defined on a compact set $M$. It follows that $H_{N}$ actually achieves its minimum on $M$. Denote this minimum by $\left(\alpha_{1}(N), \ldots, \alpha_{N}(N)\right)$ and the corresponding function

$$
\Psi_{N}\left(\alpha_{1}, \ldots, \alpha_{N}\right)=\sum_{n=1}^{N} \alpha_{n} \sin (n \theta) .
$$

It remains to find the system of algebraic equations satisfied by $\left(\alpha_{1}, \ldots, \alpha_{N}\right)$ when $\lambda>\Lambda$. For this purpose, note that equation (3.6) implies that at the minimum, which must be in the interior of $M$ since $H_{N}$ is zero on the boundary,

$$
d H_{N}=\sum_{n=1}^{N}\left(n^{2} \alpha_{n}-\frac{2 \lambda}{\pi} \int_{0}^{\pi} \sin \left(\Psi_{N}\right) \sin (n \theta) d \theta\right) d a_{n}=0 .
$$

The variations $d a_{n}$ are not arbitrary since (3.5) implies

$$
d C_{N}=\sum_{n=1}^{N}\left(\int_{0}^{\pi}\left(\sin \left(\Psi_{N}+\theta\right) \sin (n \theta) d \theta\right)\right) d a_{n}=0 .
$$

Equivalently,

$$
\begin{aligned}
d H_{N}+\mu_{N} d C_{N}=\sum_{n=1}^{N}( & n^{2} \alpha_{n}-\frac{2 \lambda}{\pi} \int_{0}^{\pi} \sin \left(\Psi_{N}\right) \sin n \theta d \theta \\
& \left.\quad+\frac{2 \mu_{N}}{\pi} \int_{0}^{\pi} \sin \left(\Psi_{N}+\theta\right) \sin n \theta d \theta\right) d a_{n} \\
= & 0
\end{aligned}
$$

for all admissible variations $d a_{n}$. Assume there is a value of $n$, say $n=k$, where $1 \leq k \leq N$ such that

$$
\int_{0}^{\pi} \sin \left(\Psi_{N}+\theta\right) \sin k \theta d \theta \neq 0
$$

In this case we can choose

$$
\mu_{N}=\frac{-(\pi / 2)\left(k^{2} \alpha_{k}-(2 \lambda / \pi) \int_{0}^{\pi} \sin \left(\Psi_{N}\right) \sin \theta d \theta\right)}{\left(\int_{0}^{\pi} \sin \left(\psi_{N}+\theta\right) \sin k \theta d \theta\right)},
$$

i.e., we choose $\mu_{N}$ so that the coefficient of $d \alpha_{k}$ in equation (3.15) vanishes. The variations $d a_{n}$ with $n \neq k$ are arbitrary; thus, for this choice of $\mu_{N}$, all the coefficients of $d a_{n}$ in (3.15) vanish. The numbers $\alpha_{1}, \ldots, \alpha_{N}$ satisfy

$$
n^{2} \alpha_{n}-\frac{2 \lambda}{\pi} \int_{0}^{\pi} \sin \left(\Psi_{N}\right) \sin n \theta d \theta+\frac{2 \mu_{N}}{\pi} \int_{0}^{\pi} \sin \left(\Psi_{N}+\theta\right) \sin n \theta d \theta=0
$$

where $\Psi_{N}$ is given by (3.12) and $n=1, \ldots, N$. The vector $\left(\alpha_{1}(N), \ldots, \alpha_{N}(N)\right)$ does not vanish if $\lambda>\Lambda$ (cf. (3.10)) since the minimum of $H_{N}$ is negative. Thus, we have proven the existence of a nontrivial solution to the finite system (3.4) satisfying 
the constraint (3.5), if we can verify (3.16) for some integer $k$. We will prove the following:

LEMMA 3.1. There exists a subsequence $\left(\alpha_{1}\left(K_{i}\right), \ldots, \alpha_{K_{i}}\left(K_{i}\right)\right)$ and a fixed integer $k$ with the property that

$$
\int_{0}^{\pi} \sin \left(\Psi_{K_{i}}+\theta\right) \sin k \theta d \theta \neq 0
$$

when $K_{i}$ is sufficiently large.

Proof. In order to simplify the notation, define $\alpha_{n}(N)=0$ for $n>N$. In this notation, it follows from (3.11) that

$$
\sum_{n=1}^{\infty} n^{2} \alpha_{n}(N)^{2} \leq 8 \lambda .
$$

The inequality (3.20) implies that $\alpha_{1}(N)$ is bounded independent of $N$, and thus there exists a subsequence $\alpha_{1}\left(N_{1}\right)$ which converges to a value $\alpha_{1}^{*}$. Similarly, $\alpha_{2}\left(N_{1}\right)$ is bounded independent of $N$ so that we can choose a subsequence of $\alpha_{2}\left(N_{1}\right)$, say $\alpha_{2}\left(N_{2}\right)$, which converges to a value $\alpha_{2}^{*}$. Continuing this procedure, we construct an infinite vector $\left(\alpha_{1}^{*}, \alpha_{2}^{*}, \ldots, \alpha_{n}^{*}, \ldots\right)$, where

$$
\sum_{n=1}^{\infty} n^{2}\left(\alpha_{n}^{*}\right)^{2} \leq 8 \lambda .
$$

We note that the diagonal sequence $\alpha_{n}\left(N_{N}\right) \rightarrow \alpha_{n}^{*}$ for every $n$ as $N_{N} \rightarrow \infty$. Define the two functions $\Psi_{N_{N}}$ and $\Psi^{*}$ by

$$
\Psi_{N_{N}}=\sum_{n=1}^{\infty} \alpha_{n}\left(N_{N}\right) \sin n \theta
$$

$\left(\right.$ recall $\alpha_{n}\left(N_{N}\right)=0$ if $\left.n>N_{N}\right)$ and

$$
\Psi^{*}=\sum_{n=1}^{\infty} \alpha_{n}^{*} \sin n \theta .
$$

The functions $\Psi_{N_{N}} \rightarrow \Psi^{*}$ uniformly as $N \rightarrow \infty$. This fact follows from the inequality

$$
\begin{aligned}
\left|\Psi_{N_{N}}-\Psi^{*}\right| \leq & \sum_{n=1}^{L}\left|\alpha_{n}\left(N_{N}\right)-\alpha_{n}^{*}\right|+\sum_{n=L+1}^{\infty}\left(\left|\alpha_{n}\left(N_{N}\right)\right|+\left|\alpha_{n}^{*}\right|\right) \\
\leq & \sum_{n=1}^{L}\left|\alpha_{n}\left(N_{N}\right)-\alpha_{n}^{*}\right| \\
& +\left(\left(\sum_{n=L+1}^{\infty} n^{2} \alpha_{n}\left(N_{N}\right)^{2}\right)^{1 / 2}+\left(\sum_{n=L+1}^{\infty} n^{2}\left(\alpha_{n}^{*}\right)^{2}\right)^{1 / 2}\right)\left(\sum_{n=L+1}^{\infty} \frac{1}{n^{2}}\right)^{1 / 2} \\
\leq & \sum_{n=1}^{L}\left|\alpha_{n}\left(N_{N}\right)-\alpha_{n}^{*}\right|+2 \sqrt{8 \lambda}\left(\sum_{n=L+1}^{\infty} \frac{1}{n^{2}}\right)^{1 / 2} .
\end{aligned}
$$


By first choosing $L$ and then choosing $N_{N}$ the right side of (3.24) can be made arbitrarily small, which guarantees the uniform convergence of $\Psi_{N_{N}}$ to $\Psi^{*}$. We also note that $\Psi^{*}$ is continuous and $\Psi^{*} \neq 0$ since

$$
H_{N_{N}}\left(\alpha_{1}\left(N_{N}\right), \ldots, \alpha_{N_{N}}\left(N_{N}\right)\right) \leq H_{N_{N}}\left(j_{11}, 0, \ldots, 0\right)<0 .
$$

The function $\Psi_{N_{N}}$ satisfies the condition

$$
\int_{0}^{\pi} \cos \left(\Psi_{N_{N}}+\theta\right) d \theta=0
$$

Taking the limit as $N_{N} \rightarrow \infty$ we find

$$
\int_{0}^{\pi} \cos \left(\Psi^{*}+\theta\right) d \theta=0
$$

$\Psi^{*}$ is a continuous function and $\Psi^{*}(0)=\Psi^{*}(\pi)=0$. Therefore, $\Psi^{*}+\theta \not \equiv 0$. Equivalently, $\sin \left(\Psi^{*}+\theta\right) \not \equiv 0$. We conclude that there exists some value of $k$ such that

$$
\int_{0}^{\pi} \sin \left(\Psi^{*}+\theta\right) \sin k \theta d \theta \neq 0
$$

Since $\Psi_{N_{N}} \rightarrow \Psi^{*}$ uniformly, it follows that when $N_{N}$ is sufficiently large,

$$
\int_{0}^{\pi} \sin \left(\Psi_{N_{N}}+\theta\right) \sin k \theta d \theta \neq 0 \text {. Q.E.D. }
$$

In the proof of the lemma, we have constructed a sequence of numbers $\left(\alpha_{1}\left(N_{N}\right)\right.$, $\left.\alpha_{2}\left(N_{N}\right), \ldots\right)$ with the following properties:

$$
\begin{aligned}
& n^{2} \alpha_{n}\left(N_{N}\right)-\frac{2 \lambda}{\pi} \int_{0}^{\pi} \sin \left(\Psi_{N_{N}}\right) \sin n \theta d \theta \\
& \quad+\frac{2 \mu_{N_{N}}}{\pi} \int_{0}^{\pi} \sin \left(\Psi_{N_{N}}+\theta\right) \sin n \theta d \theta=0
\end{aligned}
$$

for $1 \leq n \leq N_{N}$,

$$
\begin{gathered}
\int_{0}^{\pi} \sin \left(\Psi_{N_{N}}+\theta\right) \sin k \theta d \theta \neq 0, \\
\mu_{N_{N}}=-\frac{\pi}{2}\left(\frac{k^{2} \alpha_{k}\left(N_{N}\right)-(2 \lambda / \pi) \int_{0}^{\pi} \sin \left(\Psi_{N_{N}}\right) \sin k \theta d \theta}{\int_{0}^{\pi} \sin \left(\Psi_{N_{N}}+\theta\right) \sin k \theta d \theta}\right), \\
\int_{0}^{\pi} \cos \left(\Psi_{N_{N}}+\theta\right) d \theta=0 .
\end{gathered}
$$

Equations (3.30)-(3.33) hold when $N_{N}$ is sufficiently large. Passing to the limit as $N_{N} \rightarrow \infty$, we find that the members $\left(\alpha_{1}^{*}, \alpha_{2}^{*}, \ldots\right)$ satisfy

$$
n^{2} \alpha_{n}^{*}-\frac{2 \lambda}{\pi} \int_{0}^{\pi} \sin \left(\Psi^{*}\right) \sin n \theta d \theta+\frac{2 \mu}{\pi} \int_{0}^{\pi} \sin \left(\Psi^{*}+\theta\right) \sin n \theta d \theta=0
$$

for $n=1,2, \ldots$,

$$
\mu=-\frac{\pi}{2}\left(\frac{k^{2} \alpha_{k}^{*}-(2 \lambda / \pi) \int_{0}^{\pi} \sin \left(\Psi^{*}\right) \sin k \theta d \theta}{\int_{0}^{\pi} \sin \left(\Psi^{*}+\theta\right) \sin k \theta d \theta}\right),
$$




$$
\int_{0}^{\pi} \cos \left(\Psi^{*}+\theta\right) d \theta=0
$$

where $\Psi^{*}$ is given by (3.23). In particular, the values $\alpha_{1}^{*}, \alpha_{2}^{*}, \ldots$ satisfy the infinite system (3.3) and the constraint (3.36).

It remains to show that $\Psi^{*}$ is a solution of (1.1). For this purpose, consider the integral equation

$$
\Psi=\int_{0}^{\pi} G(\theta, \phi) f(\psi(\phi)) d \phi,
$$

where

$$
G(\theta, \phi)= \begin{cases}\frac{\phi \theta}{\pi}-\phi, & 0 \leq \phi \leq \theta, \\ \frac{\phi \theta}{\pi}-\theta, & \theta \leq \phi \leq \pi,\end{cases}
$$

and

$$
f(\psi(\phi))=-\lambda \sin \psi+\mu \sin (\psi+\phi) .
$$

Placing $\Psi$ in (3.37), it follows that

$$
\Psi^{*}-\int_{0}^{\pi} G(\theta, \phi) f\left(\Psi^{*}(\phi)\right) d \phi=h(\theta),
$$

where $h(\theta)$ is continuous. Multiplying by $\sin n \theta$ and integrating from $\theta=0$ to $\theta=\pi$, we find

$$
\begin{aligned}
\int_{0}^{\pi} h(\theta) \sin n \theta d \theta= & \frac{\pi}{2} \alpha_{n}^{*}-\frac{\lambda}{n^{2}} \int_{0}^{\pi} \sin \left(\Psi^{*}\right) \sin n \theta d \theta \\
& +\frac{\mu}{n^{2}} \int_{0}^{\pi} \sin \left(\Psi^{*}+\theta\right) \sin n \theta d \theta .
\end{aligned}
$$

The right side of (3.41) vanishes (cf. (3.34)). We conclude that $h(\theta) \equiv 0$, i.e.,

$$
\Psi^{*}=\int_{0}^{\pi} G(\theta, \phi) f\left(\Psi^{*}(\phi)\right) d \phi .
$$

It is an immediate consequence that $\Psi^{*}$ is twice differentiable and satisfies (1.1).

In the preceding remarks, we have shown that if $\lambda>\Lambda$, the problem (1.1), (1.2), and (1.3) has a nontrivial solution. Moreover, it is an immediate consequence of the development in this section that the energy of this solution is negative (cf. (3.9) and $(3.10))$, i.e.,

$$
H=\frac{\pi}{2} \int_{0}^{\pi}\left(\frac{\left(\psi^{\prime}\right)^{2}}{2}+\lambda(\cos \psi-1)\right) d \theta
$$

is negative. However, if $\lambda<1$ this is not possible since if $\lambda<0$ it is clear that $H \geq 0$ and if $0 \leq \lambda<1$ we have

$$
\begin{aligned}
H & =\frac{\pi}{2} \int_{0}^{\pi}\left(\frac{\left(\psi^{\prime}\right)^{2}}{2}-\lambda \frac{\psi^{2}}{2}\right) d \theta+\frac{\pi}{2} \lambda \int_{0}^{\pi}\left(\frac{\psi^{2}}{2}+(\cos \psi-1)\right) d \theta \\
& \geq \frac{\pi}{2} \int_{0}^{\pi}\left(\frac{\left(\psi^{\prime}\right)^{2}}{2}-\lambda \frac{\psi^{2}}{2}\right) d \theta
\end{aligned}
$$


The fact that the right side of (3.44) is nonnegative follows from the minimum characterization of eigenvalues. We conclude that if $\lambda<1$ the minimum energy solution is the trivial solution.

4. Numerical results. Equation (1.1) with boundary conditions (1.3) was solved numerically by "shooting" (cf. [7]), both for $\mu=0$ and $\mu \neq 0$. If $\mu=0$, equation (1.1) is the ordinary elastica equation (cf. [10]). If $\lambda>4$, this equation has nontrivial solutions which satisfy (1.2). If $\lambda \leq 4$, the only solution satisfying (1.1) with $\mu=0$, the boundary condition (1.3), and the condition (1.2) is $\psi \equiv 0$. For the nontrivial solutions, the form of the compressed ring agrees with the results described in [1]. Various possible equilibrium shapes for the buckled ring are given in Figs. 4.2-4, 4.3-7, and 4.3-8. Although they are not pictured, other solutions for larger values of $\lambda$ were also computed. In fact all the figures given in [1] were reproduced. These figures all have at least two axes of symmetry. As was indicated in the introduction, the actual solutions which are found experimentally have only one axis of symmetry:

The solutions with one axis of symmetry occur when $\mu \neq 0$. In Sec. 3, we saw that there were nontrivial solutions of (1.1), (1.2), and (1.3) for values of $\lambda<4$. These solutions were also found numerically. Equation (1.1) with boundary conditions (1.3) was solved by shooting for increasing values of $\mu$. The integral (1.2) was evaluated numerically for each $\mu$ until it changed sign. The appropriate value of $\mu$ which makes the integral vanish was obtained by successively reducing the size of the interval over which the integral changed sign.

In Fig. 4.1, we have plotted a bifurcation diagram for the circular elastica for $1 \leq \lambda \leq 7$. As a measure of deformation, we have plotted $-w(\pi) / a$ on the $y$-axis. The curve branching from $\lambda=4$ is the curve corresponding to the solutions with two axes of symmetry discussed in [1] and [2]. However, there is another parabola-like curve which does not bifurcate from the $\lambda$ axis. This curve corresponds to solutions with $\mu \neq 0$. The solutions on this curve have one axis of symmetry and exist for $\lambda<4$. In Sec. 3, we proved the existence of solutions of $\lambda>\Lambda_{0} \approx 2.62$. The

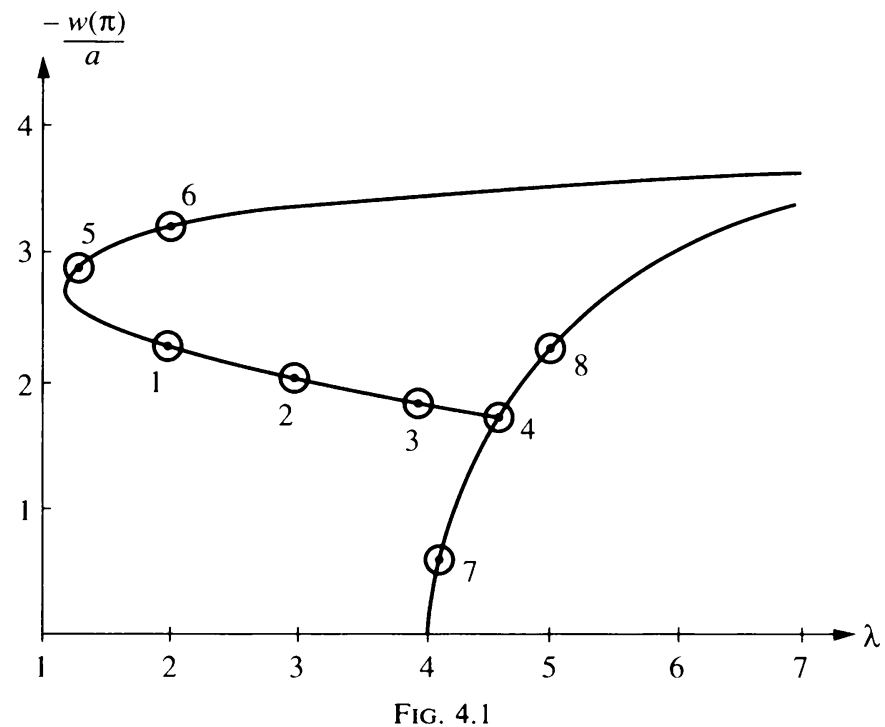

FIG. 4.1 

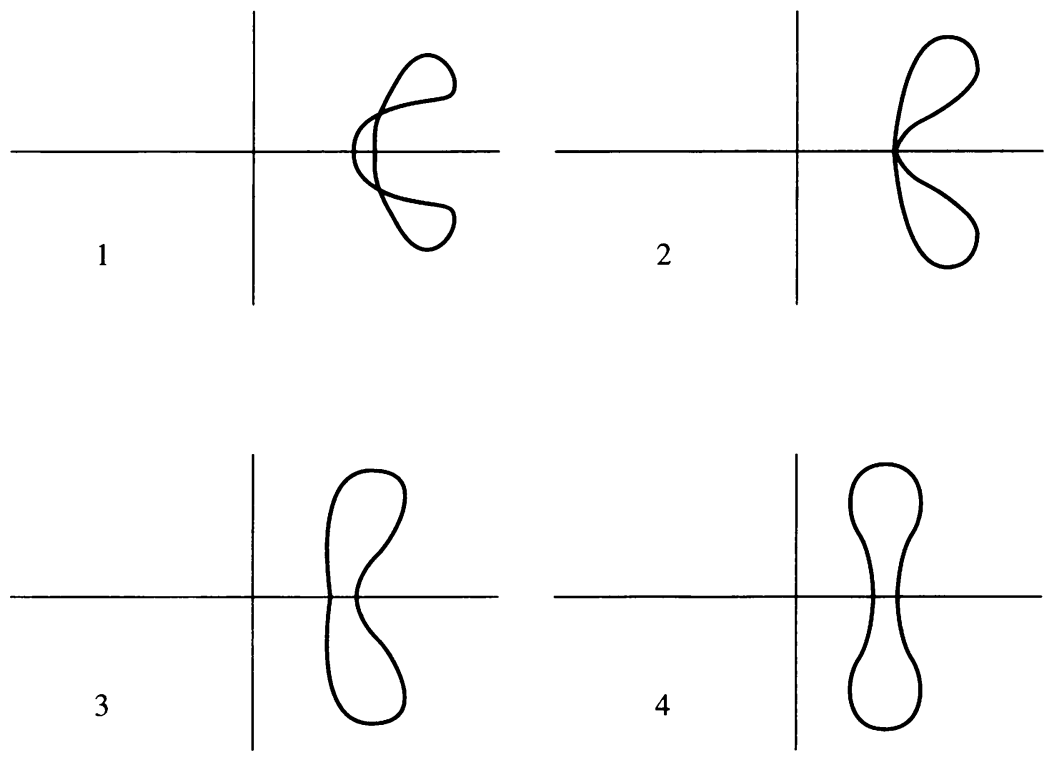

FIG. 4.2
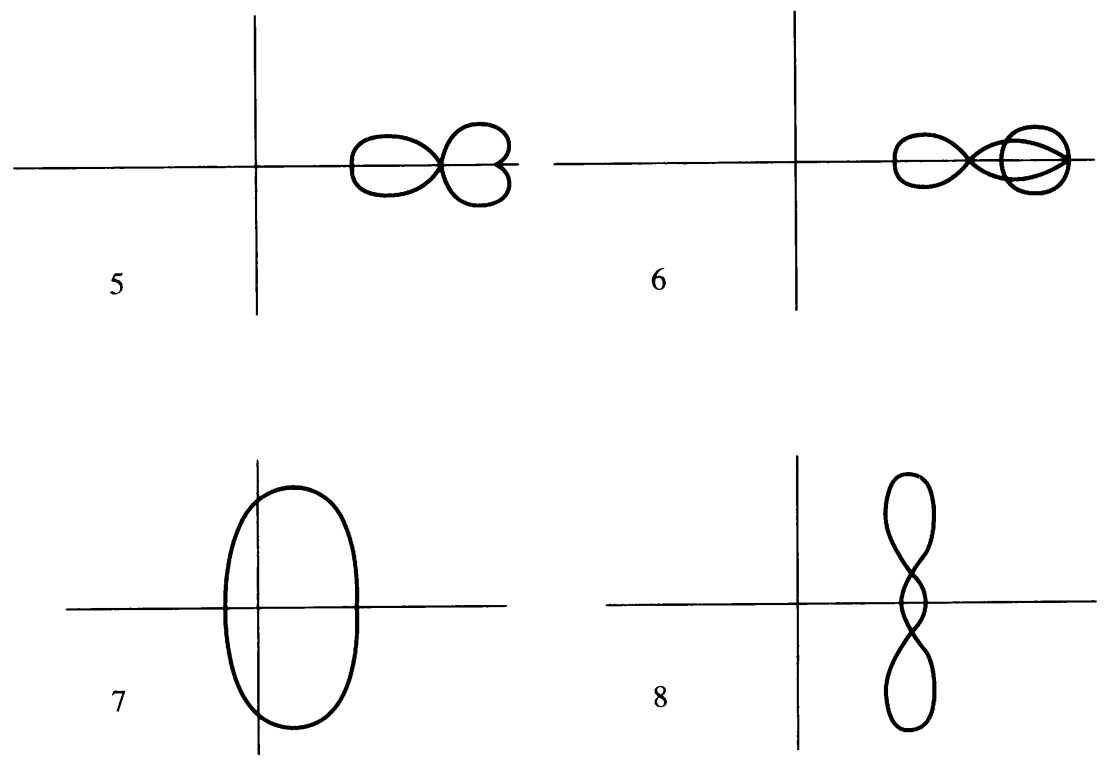

FIG. 4.3

numerical evidence indicates that solutions exist for even smaller values of $\lambda$. The vertex of the parabola-like curve in Fig. 4.1 occurs for $\lambda \approx 1.2$.

In Fig. 4.1, we have numbered various points on the bifurcation curve. These numbers correspond to those given in Figs. 4.2 and 4.3. The shapes with one axes of symmetry given in 4.2-2 and 4.2-3 actually agree closely with the shapes one obtains experimentally. Evidently, as the force is increased the ring begins to develop 
a second dip. When $\lambda$ is sufficiently large $(\lambda \equiv 4.6)$, the slope has two axes of symmetry.

Figures 4.2-1, 4.3-5, 4.3-6, 4.3-8 actually indicate that the ring passes through itself. This of course would not happen in practice. The actual shape of the ring in these cases would be affected by the fact that two different sections of the ring are in contact. The implication is that catastrophic buckling may occur at pressures considerably less than the lowest eigenpressure predicted by the linear theory.

5. Appendix. Derivation of the equations. We consider a circular ring whose radius, measured to the center line, is $a$. Before deformation the position of a point on the center line is given by

$$
\bar{R}=a \hat{e}_{r}
$$

( $\hat{e}_{r}$ and $\hat{e}_{\theta}$ will denote unit vectors in polar coordinates). After deformation this point has a new position,

$$
\bar{R}^{*}=(a+w) \hat{e}_{r}+u \hat{e}_{\theta} .
$$

The functions $w$ and $u$ depend on the polar angle $\theta$. The arc-length $d s$ of the undeformed center line is given by

$$
d s^{2}=d \bar{R} \cdot d \bar{R}=a^{2} d \theta^{2},
$$

while the arc-length $d s^{*}$ of the deformed center line is given by

$$
\left(d s^{*}\right)^{2}=d \bar{R}^{*} \cdot d \bar{R}^{*}=\left[\left(1+\frac{w+u^{\prime}}{a}\right)^{2}+\left(\frac{w^{\prime}-u}{a}\right)^{2}\right] a^{2} d \theta^{2}
$$

$\left({ }^{\prime}=d / d \theta\right)$. The strain $\varepsilon$ of the center line is defined by

$$
\left(d s^{*}\right)^{2}-d s^{2}=2 e a^{2} d \theta^{2}=2 e d s^{2}
$$

so that

$$
e=\frac{w+u^{\prime}}{a}+\frac{1}{2}\left(\frac{w+u^{\prime}}{a}\right)^{2}+\frac{1}{2}\left(\frac{w^{\prime}-u}{a}\right)^{2} .
$$

It is a consequence of (5.5) that

$$
\frac{d s^{*}}{d s}=\sqrt{1+2 e}
$$

or, equivalently,

$$
\frac{d s^{*}}{d \theta}=a \sqrt{1+2 e} .
$$

The unit tangent to the deformed center line is given by

$$
\hat{t}=\frac{\bar{R}^{* \prime}}{\left|\bar{R}^{* \prime}\right|}=\frac{\left(w^{\prime}-u\right) \hat{e}_{r}+\left(a+w+u^{\prime}\right) \hat{e}_{\theta}}{a \sqrt{1+2 e}}
$$

and the unit normal is

$$
\hat{n}=\frac{-\left(a+w+u^{\prime}\right) \hat{e}_{r}+\left(w^{\prime}-u\right) \hat{e}_{\theta}}{a \sqrt{1+2 e}} .
$$


Before deformation, the position of a point off the center line can be written

$$
\bar{X}=(a-r) \hat{e}_{r}=\bar{R}-r \hat{e}_{r} .
$$

In order to describe the position of the point after deformation, we will use the assumptions von Karman introduced in his study of plates and shells (cf. [4]). In particular, we assume (i) a normal to the center line before deformation remains normal after deformation, and (ii) a point at a distance $r$ from the center line before deformation remains a distance $r$ after deformation. Combining these remarks with (5.11), we find that after deformation the position is given by

$$
\bar{X}^{*}=(a+w) \hat{e}_{r}+u \hat{e}_{\theta}+r \hat{n}=\bar{R}^{*}+r \hat{n} .
$$

Equivalently (cf. (5.10)),

$$
\begin{aligned}
\bar{X}^{*}= & \left(a+w-r \frac{a+w+u^{\prime}}{a \sqrt{1+2 e}} \hat{e}_{r}\right. \\
& +\left(u+r \frac{w+u^{\prime}}{a \sqrt{1+2 e}}\right) \hat{e}_{\theta} .
\end{aligned}
$$

It is convenient to introduce a new variable. Define an angle $\psi$ by

$$
\begin{aligned}
-\sin \psi & =\frac{w^{\prime}-u}{a \sqrt{1+2 e}}, \\
\cos \psi & =\frac{a+w+u^{\prime}}{a \sqrt{1+2 e}}
\end{aligned}
$$

so that the unit tangent to the deformed center line is

$$
\hat{t}=-\sin \psi \hat{e}_{r}+\cos \psi \hat{e}_{\theta} .
$$

Geometrically, the variable $\psi$ is the change in angle of the tangent to the middle line before and after deformation. Similarly the unit normal is given by

$$
\hat{n}=-\left(\cos \psi \hat{e}_{r}+\sin \psi \hat{e}_{\theta}\right) \text {. }
$$

The Frenet-Serret equations (cf. [8]) imply that

$$
\frac{d \hat{t}}{d s^{*}}=K \hat{n},
$$

where $K$ is the curvature. The curvature is easily computed from (5.15), i.e.,

$$
\frac{d t}{d s^{*}}=\left[\frac{d}{d \theta}\left(-\sin \psi \hat{e}_{r}+\cos \psi \hat{e}_{\theta}\right)\right] \frac{d \theta}{d s^{*}}=\frac{\psi^{\prime}+1}{a \sqrt{1+2 e}} \hat{n} .
$$

Therefore,

$$
K=\frac{\psi^{\prime}+1}{a \sqrt{1+2 e}} .
$$

In order to compute the strains off the center line, we note that

$$
\begin{aligned}
d \bar{X}^{*}=\frac{\partial \bar{X}^{*}}{\partial \theta} d \theta+\frac{\partial \bar{X}^{*}}{\partial r} d r & =\left(\frac{d \bar{R}^{*}}{d \theta}+r \frac{d \hat{n}}{d \theta}\right) d \theta+d r \hat{n} \\
& =\left(\frac{d \bar{R}^{*}}{d s^{*}}(a \sqrt{1+2 e})+r \frac{d \hat{n}}{d \theta}\right) d \theta+d r \hat{n}
\end{aligned}
$$


Observing that

and

$$
\frac{d \bar{R}^{*}}{d s^{*}}=\hat{t}
$$

we find that

$$
\frac{d \hat{n}}{d \theta}=-\left(\psi^{\prime}+1\right) \hat{t}
$$

$$
\begin{aligned}
d \bar{X}^{*} & =\left[a \sqrt{1+2 e}-r\left(\psi^{\prime}+1\right)\right] d \theta \hat{t}+d r \hat{n} \\
& =a \sqrt{1+2 e}(1-r K) d \theta \hat{t}+d r \hat{n} .
\end{aligned}
$$

Similarly, we compute $d \bar{X}$ to show

$$
d \bar{X}=(a-r) d \theta \hat{e}_{\theta}-d r \hat{e}_{r}
$$

so that

$$
\begin{aligned}
d \bar{X}^{*} \cdot d \bar{X}^{*}-d \bar{X} \cdot d \bar{X} & =\left[\frac{a^{2}}{(a-r)^{2}}(1+2 e)(1-r K)^{2}-1\right](a-r)^{2} d \theta^{2} \\
& =2 \varepsilon(a-r)^{2} d \theta^{2}
\end{aligned}
$$

It follows from (5.25) that the strain $\varepsilon$ at any point in the ring is given by

$$
\varepsilon=\frac{1}{2}\left[\frac{a^{2}}{(a-r)^{2}}(1+2 e)(1-r K)^{2}-1\right],
$$

or, equivalently,

$$
\sqrt{1+2 \varepsilon}=\frac{a}{a-r} \sqrt{1+2 e}(1-r K) .
$$

In this paper, we assume the ring is inextensible, i.e., we assume the center line strain $e=0$. In this case the curvature $K$ is given by (cf. 5.19),

$$
K=\frac{\psi^{\prime}+1}{a} .
$$

The displacement $u$ and $w$ satisfy the ordinary differential equations (cf. (5.14))

$$
\begin{gathered}
\frac{w^{\prime}-u}{a}=-\sin \psi, \\
1+\frac{w+u^{\prime}}{a}=\cos \psi .
\end{gathered}
$$

In addition we assume $\varepsilon$ is small. Thus equation (5.27) implies

$$
\varepsilon=-\frac{r}{a-r} \psi^{\prime} .
$$

Since the strains are small, the strain energy is given by

$$
\mathscr{E}=\int_{A} \int\left(\int_{-\pi}^{\pi}\left(\frac{E}{2} \varepsilon^{2} a d \theta\right)\right) d A,
$$

when $A$ is the area of a cross section of an element of the ring. It follows from (5.30) that the strain energy can be written

$$
\mathscr{E}=\int_{-\pi}^{\pi} \frac{E I}{2}\left(\psi^{\prime}\right)^{2} a d \theta
$$

where $I$ is given by (1.5). 
It will be assumed that the ring is acted on by a constant force $P$ per unit length in the direction normal to the undeformed ring, i.e., in the direction $\hat{e}_{r}$. The work done by this force will be taken to be

$$
W=-\int_{-\pi}^{\pi} P w a d \theta
$$

This expression is the one used by Stoker [4] and is a consequence of the assumption that the work due to circumferential displacement is small compared to the work due to radial displacement.

For our purposes it is convenient to rewrite the work integral (5.33) in terms of $\psi$. Eliminating $u$ from (5.29), we find that

$$
w^{\prime \prime}+w=a\left(\cos \psi-\psi^{\prime} \cos \psi-1\right) .
$$

Once $w$ is determined, $u$ is given by

$$
u=w^{\prime}+a \sin \psi .
$$

The appropriate boundary conditions on $u, w$, and $\psi$ are the periodicity conditions

$$
\begin{aligned}
u(-\pi) & =u(\pi), & u^{\prime}(-\pi) & =u^{\prime}(\pi), \\
w(-\pi) & =w(\pi), & w^{\prime}(-\pi) & =w^{\prime}(\pi), \\
\psi(-\pi) & =\psi(\pi), & \psi^{\prime}(-\pi) & =\psi^{\prime}(\pi) .
\end{aligned}
$$

It is a consequence of the Fredholm alternative theorem (cf. [9]) that a necessary and sufficient condition for (5.34) with boundary conditions $(5.36 \mathrm{~b})$ to have a solution is

$$
\begin{aligned}
\int_{-\pi}^{\pi} & \sin \theta\left(\cos \psi-\psi^{\prime} \cos \psi-1\right) d \theta \\
& =\int_{-\pi}^{\pi} \cos \theta\left(\cos \psi-\psi^{\prime} \cos \psi-1\right) d \theta \\
& =0 .
\end{aligned}
$$

After an integration by parts, the constraints (5.37) have the form

$$
\int_{-\pi}^{\pi} \sin (\psi+\theta) d \theta=\int_{-\pi}^{\pi} \cos (\psi+\theta) d \theta=0 .
$$

Assuming $\psi$ satisfies (5.38), there is no difficulty in showing that

$$
w=-a(1+\cos \theta)-a \cos \theta \int_{-\pi}^{\theta} \sin (\psi+\zeta) d \zeta+a \sin \theta \int_{-\pi}^{\theta} \cos (\psi+\zeta) d \zeta .
$$

Of course $w$ is only determined up to a linear combination of $\sin \theta$ and $\cos \theta$. However, these terms will have no effect on the work. It follows from (5.35) and (5.39) that

$$
u=a \sin \theta+a \sin \theta \int_{-\pi}^{\theta} \sin (\psi+\zeta) d \zeta+a \cos \theta \int_{-\pi}^{\theta} \cos (\psi+\zeta) d \zeta
$$


Combining (5.33) and (5.39) causes the work to become

$$
W=-P a^{2} \int_{-\pi}^{\pi}\left[-1-\cos \theta \int_{-\pi}^{\theta} \sin (\psi+\zeta) d \zeta+\sin \theta \int_{-\pi}^{\theta} \cos (\psi+\zeta) d \zeta\right] d \theta
$$

After an integration by parts, the constraints (5.38) imply

$$
\begin{aligned}
W & =-P a^{2} \int_{-\pi}^{\pi}[\sin (\psi+\theta) \sin \theta+\cos (\psi+\theta) \cos \theta-1] d \theta \\
& =-P a^{2} \int_{-\pi}^{\pi}[\cos \psi-1] d \theta .
\end{aligned}
$$

The equilibria of the circular ring are solutions of the problem

$$
\delta(\mathscr{E}-W)=\delta \int_{-\pi}^{\pi}\left(\frac{E I}{2}\left(\psi^{\prime}\right)^{2}+P a(\cos \psi-1)\right) a d \theta=0,
$$

subject to the boundary conditions (5.36) and constraints (5.38). It follows from standard arguments (cf. [9]) that $\psi$ must satisfy the ordinary differential equation

$$
\frac{d^{2} \psi}{d \theta}+\lambda \sin \psi-\mu_{1} \sin (\psi+\theta)+\mu_{2} \cos (\psi+\theta)=0,
$$

where $\lambda$ is given by (1.4), $\psi$ is to satisfy the boundary conditions (5.36), and $\mu_{1}$ and $\mu_{2}$ are to be chosen to satisfy the constraints (5.38).

This problem can be simplified. Assume we solve the problem (1.1) with constraint (1.2) and boundary condition (1.3). Extend the definition of $\psi$ to the interval $-\pi \leq \theta \leq 0$ by defining $\psi$ as the odd extension on this interval, i.e., $\psi(\theta)=-\psi(-\theta)$ for $-\pi \leq \theta \leq 0$. This function will satisfy equation (5.44), with $\mu_{1}=\mu$ and $\mu_{2}=0$. In addition, it will satisfy the constraints (5.38) and boundary conditions (5.36). Thus, it suffices to study the problem (1.1), (1.2), and (1.3).

(An anonymous reviewer writes ".... a purely Newtonian derivation of the governing equations... reveals that the nonautonomous 'forcing' term in (1.1) represents the combined contribution of the axial and shear forces to the bending moment at section ' $s$ ' in a given nonradial $(\mu \neq 0)$ state.")

\section{REFERENCES}

[1] I. Tadjbakhsh and F. Odeh, Equilibrium states of elastic rings, J. Math. and Appl. 18, 59-74 (1967)

[2] S. S. Antman, A note on a paper of Tadjbakhsh and Odeh, J. Math. Anal. Appl. 21, 132-135 (1968)

[3] S. S.Antman, The shape of buckled nonlinear elastic rings, Z. angew. Math. Phys. 21, 422-438 (1970)

[4] J. J. Stoker, Nonlinear Elasticity, Gordon and Breach, New York, 1968

[5] R. W. Dickey, Nonlinear bending of circular plates, SIAM J. Appl. Math. 30, 1-9 (1976)

[6] R. W. Dickey Minimum energy solution for the spherical shell, Quart. Appl. Math. 48, 321-339 (1990)

[7] E. Isaacson and H. B. Keller, Analysis of numerical methods, Wiley, New York, 1966

[8] B. J. Struik, Lectures on classical differential geometry, Addison-Wesley, Reading, MA, 1961

[9] R. Courant and D. Hilbert, Methods of mathematical physics, vol. 1, Interscience, New York, 1953

[10] E. L. Reiss, Column buckling-an elementary example of bifurcation, Bifurcation Theory and Nonlinear Eigenvalue Problems (J. B. Keller and S. Antman eds.), W. A. Benjamin, New York, 1969 